for Advancement in Medicine, the main group that promotes this treatment, has reported that over 800,000 visits were made for chelation therapy in the US in 1997 alone.

EDTA administration is approved by the Food and Drug Administration for cases of lead or mercury poisoning, as it can bind these metals to create compounds that are excreted in the urine. But EDTA chelation is also widely used as an 'alternative' treatment for atherosclerosis. However, there are standard and well-proven ways to reduce the risks of complications of athero- sclerosis and coronary artery disease, and the worry is that if people chose an unproven treatment like chelation therapy, they might forego established treatments. Therefore, many say the trial is important. "It's a win-win situation," says Zorina Galis of Emory University. "If it is shown to be ineffective or harmful, hopefully doctors will stop using it. If it works, it could save lives."

EDTA is a fairly non-specific trap for non-iron metals and thus could influence the function of a myriad of molecules in the body. One of the possible ways that it could affect heart function is by removing from the circulation loose metal ions involved in oxidation, which can damage vessel walls, or by affecting the function of enzymes that require metal ions to be active, although several other mechanisms have been proposed. Galis has shown that in vitro EDTA can inhibit the function of matrix metalloproteinases, enzymes that contribute to the formation of atherosclerotic plaques. "There are reasons for which you could argue this treatment could work," she says.

\section{Laura Bonetta, Bethesda}

\title{
Nuclear receptor community goes virtual
}

In five or so years' time, it may no longer be pertinent for biomedical researchers to state which university department they belong to, but rather for them to describe their affiliation in terms of which global consortium they are a member of. This prediction is based on the rapidly growing number of teams of international scientists who are exploiting new computing and communications systems to consolidate their research community into a single web-centered entity.

The newest example of this strategy is the Functional Atlas of Orphan Nuclear Receptors, created last month with US \$3 million annual funding from the National Institute of Diabetes and Digestive and Kidney Diseases (NIDDK).

At the hub of this Consortium is a core of five centers: Baylor College of Medicine in Houston; the Salk Institute in La Jolla; University of Texas Southwestern Medical Center at Dallas; the University of Pennsylvania School of Medicine in Philadelphia and Duke University Medical Center, North Carolina. The initiative is lead by nuclear receptor (NR) experts Bert O'Malley of Baylor and Ron Evans of the Salk Institute who acknowledge, "one of the weak links... in our field remains the relatively ineffective lines of communication between institutes and laboratories worldwide." They have recognized that technological 'revolutions' in bioinformatics, personal computing, genomics and proteomics "offer opportunities to tackle our goals at a different level and pace than was previously possible."

NRs are transcription factors that regulate the expression of target genes involved in development, metabolism and reproduction. Several classes of receptor belonging to this so-called 'superfamily' have been identified as responding to ligands such as steroids, retinoids and thyroids. No

such ligands have yet been identified for a subgroup of NRs (for example, peroxisome proliferator-activated receptor (PPAR), lipid receptors, COUP-TFI/II and SF-1), hence these are called orphan NRs (ONRs). "It's the orphans that seem to be controlling almost all aspects of metabolism in the body, from lipid to carbohydrate metabolism and so on," says O'Malley. "This is why NIDDK is very hot in this area because it fits in with their overall goals."

NRs recruit cellular factors, or coregulators, to mediate their effects. For example, those coregulators that promote transcriptional acti-

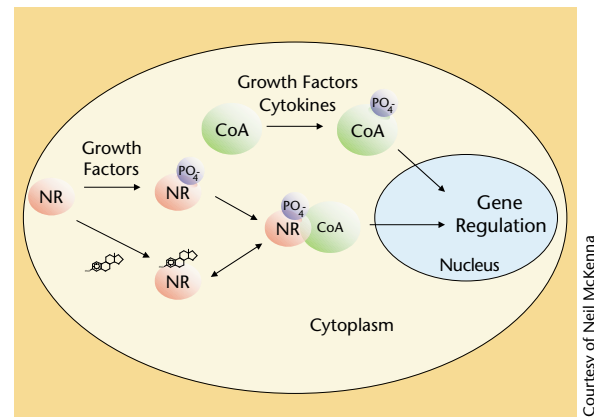

Nuclear receptors mediate the transcriptional response to a variety of lipophilic ligands, as well as certain membrane receptor signal transduction pathways. New data suggest that their recruitment of coregulators contributes to the large diversity of transcriptional response to these molecules vation are called

coactivators and those that attenuate it are corepressors. Because much is already known about NR function, the aim of the project is to build up data on ONRs more rapidly by making the Atlas the central repository for information on everything from their coregulators to target genes, ancillary proteins, activators and repressors.

But are there sufficient scientists worldwide working in this discipline to warrant the creation of a virtual global laboratory? "It's the biggest field of transcription factors," answers O'Malley, "there are 48 NR members and 30 that are orphan. There's something like 5,000 people working on the glucocorticoid receptor at some level in the world, and probably more for the estrogen receptor." The Atlas website will be op- erative in October and will contain data from the core laboratories and the general scientific population, and also a site for comments.

The ONR Atlas joins an expanding pool of similar initiatives. The best known of these are the 'glue grants' awarded by National Institute of General Medical Sciences, which fund projects such as the 5 year, \$25-million Alliance for Cellular Signaling, headed by Alfred Gilman of University of Texas Southwestern (Nature Med. 6, 1072; 2000); and the 5-year, \$37million 'inflammation of burns and trauma' project. A smaller effort is the new Myc gene website (http://www.myc-cancer-gene.org/). Likewise, Tony Pawson of Canada's Samuel Lunenfeld Research Institute heads the Biomolecular Interaction Network Database (BIND), which aims to be a comprehensive source of protein interactions that trigger chemical reactions in the body (http://www.bind.ca/).

All new sectors require service providers, and BIND's creation last year was accompanied by the launch of a non-profit bimolecular data-management organization, blueprint WORLDWIDE, which aims to be the "definitive, worldwide source for biomolecular interaction information." Perhaps scientific business cards of the future will list just a URL rather than a physical address.

Karen Birmingham, London 